\title{
Attractor bifurcation for the extended Fisher-Kolmogorov equation with periodic boundary condition
}

Qiang Zhang ${ }^{1 *}$ and Hong Luo ${ }^{2}$

\author{
"Correspondence: \\ zqcs007@163.com \\ ${ }^{1}$ College of Computer Science, Civil \\ Aviation Flight University of China, \\ Guanghan, Sichuan 618307, \\ P.R. China \\ Full list of author information is \\ available at the end of the article
}

\begin{abstract}
In this paper, we study the bifurcation and stability of solutions of the extended Fisher-Kolmogorov equation with periodic boundary condition. We prove that the system bifurcates from the trivial solution to an attractor as parameter crosses certain critical value. The topological structure of the attractor is also investigated.

MSC: 35B32; 35K35; 37G35
\end{abstract}

Keywords: extended Fisher-Kolmogorov equation; periodic boundary condition; attractor bifurcation; center manifold

\section{Introduction}

In this paper we work with the extended Fisher-Kolmogorov type equation with periodic boundary condition, which reads

$$
\left\{\begin{array}{l}
\frac{\partial u}{\partial t}=-\mu \frac{\partial^{4}}{\partial x^{4}} u+\alpha \frac{\partial^{2}}{\partial x^{2}} u+\lambda u+g(u), \quad(x, t) \in \mathbb{R} \times(0, \infty) \\
\int_{0}^{2 \pi} u(x, t) d x=0, \quad t \geq 0 \\
u(x, t)=u(x+2 k \pi, t), \quad \forall k \in \mathbb{Z}, \\
u(x, 0)=u_{0}, \quad x \in \mathbb{R},
\end{array}\right.
$$

where $u=u(x, t): \mathbb{R} \times[0, \infty) \rightarrow \mathbb{R}$ is an unknown function, $\mu>0, \alpha>0$ are constants, $\lambda \in \mathbb{R}^{+}$is the system parameter. $g(s)$ is a polynomial on $s \in \mathbb{R}$, which is given by

$$
g(s)=\sum_{k=2}^{p} a_{k} s^{k}
$$

where $2 \leq p \in \mathbb{N}$ and $a_{k}$ are given constants.

The extended Fisher-Kolmogorov (EFK) equation has been proposed as a model for phase transitions and other bistable phenomena [1-3]. It has been extensively studied during past decades. Kalies and van der Vorst [4] considered the steady-state problem; by analyzing the variational structure, they proved the existence of heteroclinic connections, which are the critical points of a certain functional. Also, by the variational method, Tersian and Chaparova [5] derived the existence of periodic and homoclinic solutions. Peletier and Troy [6] were interested in the stationary spatially periodic patterns and

\section{Springer}

C) 2013 Zhang and Luo; licensee Springer. This is an Open Access article distributed under the terms of the Creative Commons Attribution License (http://creativecommons.org/licenses/by/2.0), which permits unrestricted use, distribution, and reproduction in any medium, provided the original work is properly cited. 
showed that the structure of the solutions is enriched by increasing the coefficient of the fourth-order derivative term. The structure of the solution set was also investigated by van den Berg [7], who enumerated all the possible bounded stationary solutions provided this coefficient is small. Rottschäer and Wayne [8] showed that for every positive wavespeed there exists a traveling wave. And they also found the critical wavespeed to discriminate the monotonic solution from the oscillatory one. By an iteration procedure, Luo and Zhang [9] proved that equation (1.1) possesses a global attractor in the Sobolev space $H^{k}$ for all $k>0$ provided that $a_{p}<0$ and $p$ is an odd number. We refer the interested readers to the references in [4-9] for other results on the EFK equation; see also, among others, [10-13].

Returning to problem (1.1), our main interest in the present paper is the bifurcation and stability of solutions. By using a notion of bifurcation called attractor bifurcation developed by Ma and Wang in $[14,15]$, a nonlinear attractor bifurcation theory for this problem is established. Work on the topic of attractor bifurcation also can be seen in $[16,17]$.

The main objectives of this theory include:

(1) existence of attractor bifurcation when the system parameter crosses some critical number,

(2) dynamic stability of bifurcated solutions, and

(3) the topological structure of the bifurcated attractor.

Our main results can be summarized as follows.

1. If $\lambda \leq \mu+\alpha$, the steady state $u=0$ is locally asymptotically stable.

2. As $\lambda$ crosses $\mu+\alpha$, i.e., there exists an $\epsilon>0$ such that for any $\mu+\alpha<\lambda<\lambda+\epsilon$, system (1.1) bifurcates from the trivial solution to an attractor $\Sigma_{\lambda}$.

3. $\Sigma_{\lambda}$ is homeomorphic to $S^{1}$ and consists of exactly one cycle of steady solutions of (1.1).

Moreover, we apply this theory to a model of the population density for single-species and derive biological results.

This article is organized as follows. The preliminaries are given in Section 2. The mathematical setting is presented in Section 3. The mathematical results are given in Section 4. In Section 5 we apply mathematical results to a model of the population density for singlespecies and derive biological results. In Section 6 we discuss some existing results and compare them with ours. Finally, Section 7 is devoted to the conclusions.

\section{Preliminaries}

We begin with the definition of attractor bifurcation which was first proposed by Ma and Wang in $[14,15]$.

Let $H$ and $H_{1}$ be two Hilbert spaces, and let $H_{1} \hookrightarrow H$ be a dense and compact inclusion. We consider the following nonlinear evolution equations

$$
\left\{\begin{array}{l}
\frac{d u}{d t}=L_{\lambda} u+G(u), \\
u(0)=u_{0},
\end{array}\right.
$$

where $u:[0, \infty) \rightarrow H$ is the unknown function, $\lambda \in \mathbb{R}$ is the system parameter, and $L_{\lambda}$ : $H_{1} \rightarrow H$ are parameterized linear completely continuous fields depending continuously 
on $\lambda$, which satisfy

$$
\begin{cases}L_{\lambda}=A+B_{\lambda} & \text { a sectorial operator, } \\ A: H_{1} \rightarrow H & \text { a linear homeomorphism, } \\ B_{\lambda}: H_{1} \rightarrow H & \text { parameterized linear compact operators. }\end{cases}
$$

Since $L_{\lambda}$ is a sectorial operator which generates an analytic semigroup $S_{\lambda}(t)=\left\{e^{t L_{\lambda}}\right\}_{t \geq 0}$ for any $\lambda \in \mathbb{R}$, we can define fractional power operators $\left(-L_{\lambda}\right)^{\mu}$ for $0 \leq \mu \leq 1$ with domain $H_{\mu}=D\left(\left(-L_{\lambda}\right)^{\mu}\right)$ such that $H_{\mu_{1}} \subset H_{\mu_{2}}$ if $\mu_{1}>\mu_{2}$, and $H_{0}=H$ (see $[18,19]$ ).

In addition, we assume that the nonlinear terms $G: H_{\alpha} \rightarrow H$ for some $0 \leq \alpha<1$ are a family of parameterized $C^{r}$ bounded operators $(r \geq 1)$ such that

$$
G(u)=o\left(\|u\|_{H_{\alpha}}\right)
$$

Definition 2.1 [15] A set $\Sigma \subset H$ is called an invariant set of (2.1) if $S(t) \Sigma=\Sigma$ for any $t \geq 0$. An invariant set $\Sigma \subset H$ of (2.1) is said to be an attractor if $\Sigma$ is compact, and there exists a neighborhood of $W \subset H$ of $\Sigma$ such that for any $\varphi \in W$ we have

$$
\lim _{t \rightarrow \infty} \operatorname{dist}_{H}(u(t, \varphi), \Sigma)=0,
$$

where $\operatorname{dist}_{H}(u(t, \varphi), \Sigma)=\inf _{v \in \Sigma}\|u(t, \varphi)-v\|_{H}, \forall t \geq 0$.

Definition 2.2 [15] (1) We say that the solution to equation (2.1) bifurcates from $(u, \lambda)=$ $\left(0, \lambda_{0}\right)$ to an invariant set $\Sigma_{\lambda}$ if there exists a sequence of invariant sets $\left\{\Sigma_{\lambda_{n}}\right\}$ of (2.1) such that $0 \notin \Sigma_{\lambda_{n}}$, and

$$
\begin{aligned}
& \lim _{n \rightarrow \infty} \lambda_{n}=\lambda_{0}, \\
& \lim _{n \rightarrow \infty} \max _{v \in \Sigma_{\lambda_{n}}}\|v\|_{H}=0 .
\end{aligned}
$$

(2) If the invariant sets $\Sigma_{\lambda}$ are attractors of (2.1), then the bifurcation is called attractor bifurcation.

To prove the main result, we introduce an important theorem.

Let the eigenvalues (counting multiplicity) of $L_{\lambda}$ be given by

$$
\beta_{k}(\lambda) \in \mathbb{C} \quad(k \geq 1)
$$

and the principle of exchange of stabilities holds true:

$$
\begin{aligned}
& \operatorname{Re} \beta_{i}(\lambda) \begin{cases}<0, & \text { if } \lambda<\lambda_{0}, \\
=0, & \text { if } \lambda=\lambda_{0}(1 \leq i \leq m), \\
>0, & \text { if } \lambda>\lambda_{0},\end{cases} \\
& \operatorname{Re} \beta_{j}\left(\lambda_{0}\right)<0, \quad \forall j \geq m+1 .
\end{aligned}
$$


Let the eigenspace of $L_{\lambda}$ at $\lambda=\lambda_{0}$ be

$$
E_{0}=\bigcup_{1 \leq j \leq m} \bigcup_{k=1}^{\infty}\left\{u, v \in H_{1} \mid\left(L_{\lambda_{0}}-\beta_{j}\left(\lambda_{0}\right)\right)^{k} w=0, w=u+i v\right\}
$$

It is known that $\operatorname{dim} E_{0}=m$.

The following attractor bifurcation theorem can be found in [15].

Theorem 2.1 Let $H_{1}=H=\mathbb{R}^{n}$, conditions (2.4), (2.5) hold true, and $u=0$ is a locally asymptotically stable equilibrium point of (2.1) at $\lambda=\lambda_{0}$. Then the following assertions hold true:

(1) Equation (2.1) bifurcates from $(u, \lambda)=\left(0, \lambda_{0}\right)$ to attractors $\Sigma_{\lambda}$ for $\lambda>\lambda_{0}$, with dimension $m-1 \leq \operatorname{dim} \Sigma_{\lambda} \leq m$, which is connected as $m>1$.

(2) The attractor $\Sigma_{\lambda}$ is a limit of a sequence of m-dimensional annuli $A_{k}$ with $A_{k+1} \subset A_{k}$; especially, if $\Sigma_{\lambda}$ is a finite simplicial complex, then $\Sigma_{\lambda}$ has the homology type of the $(m-1)$-dimensional sphere $S^{m-1}$.

(3) For any $u_{\lambda} \in \Sigma_{\lambda}, u_{\lambda}$ can be expressed as

$$
u_{\lambda}=v_{\lambda}+o\left(\left\|v_{\lambda}\right\|_{H_{1}}\right), \quad v_{\lambda} \in E_{0}
$$

(4) If $u=0$ is globally asymptotically stable for (2.1) at $\lambda=\lambda_{0}$, then for any bounded open set $U \subset H$ with $0 \in U$, there is an $\epsilon>0$ such that $\lambda_{0}<\lambda<\lambda_{0}+\epsilon$, the attractor $\Sigma_{\lambda}$ attracts $U \backslash \Gamma$ in $H$, where $\Gamma$ is the stable manifold of $u=0$ with codimension $m$. In particular, if (2.1) has a global attractor for all $\lambda$ near $\lambda_{0}$, then $U=H$.

Remark 2.1 As $H_{1}$ and $H$ are infinite dimensional Hilbert spaces, if (2.1) satisfies conditions (2.2)-(2.5) and $u=0$ is a locally (global) asymptotically stable equilibrium point of (2.1) at $\lambda=\lambda_{0}$, then the assertions (1)-(4) of Theorem 2.1 hold; see [14, 15].

To get the structure of the bifurcated solutions, we introduce another theorem.

Let $v$ be a two-dimensional $C^{r}(r \geq 1)$ vector field given by

$$
v_{\lambda}(x)=\lambda x-F(x)
$$

for $x \in \mathbb{R}^{2}$. Here

$$
F(x)=F_{k}(x)+o\left(|x|^{k}\right)
$$

where $F_{k}$ is a $k$-multilinear field, which satisfies the inequality

$$
C_{1}|x|^{k+1} \leq\left\langle F_{k}(x), x\right\rangle \leq C_{2}|x|^{k+1}
$$

for some constants $0<C_{1}<C_{2}$ and $k=2 m+1, m \geq 1$.

Theorem 2.2 (Theorem 5.10 in [15]) Under conditions (2.7), (2.8), the vector field (2.6) bifurcates from $(x, \lambda)=(0,0)$ to an attractor $\Sigma_{\lambda}$ for $\lambda>0$, which is homeomorphic to $S^{1}$. Moreover, one and only one of the following conclusions is true: 
(1) $\Sigma_{\lambda}$ is a period orbit.

(2) $\Sigma_{\lambda}$ consists of infinitely many singular points.

(3) $\Sigma_{\lambda}$ contains at most $2(k+1)=4(m+1)$ singular points and has $4 N+n(N+n \geq 1)$ singular points, $2 N$ of which are saddle points, $2 N$ of which are stable node points (possibly degenerate), and $n$ of which have index zero.

\section{Mathematical setting}

Let

$$
H=L^{2}(0,2 \pi)
$$

and

$$
H_{1}=\left\{u \in H^{4}(0,2 \pi) \mid u(x+2 \pi)=u(x), \int_{0}^{2 \pi} u d x=0\right\} .
$$

We define $L_{\lambda}=A+B_{\lambda}: H_{1} \rightarrow H$ and $G: H_{1} \rightarrow H$ by

$$
\left\{\begin{array}{l}
A u=-\mu \frac{d^{4}}{d x^{4}} u+\alpha \frac{d^{2}}{d x^{2}} u, \\
B_{\lambda} u=\lambda u, \\
G(u)=g(u) .
\end{array}\right.
$$

Consequently, we have an operator equation which is equivalent to problem (1.1) as follows:

$$
\left\{\begin{array}{l}
\frac{d u}{d t}=L_{\lambda} u+G(u), \\
u(0)=u_{0} .
\end{array}\right.
$$

\section{Mathematical results}

As mentioned in the introduction, we study in this manuscript attractor bifurcation of the EFK equation under the periodic boundary condition. Then we have the following bifurcation theorem.

Theorem 4.1 For problem (1.1), if $2 a_{2}^{2}+45 \mu a_{3}+9 \alpha a_{3}<0$ is satisfied, then the following assertions hold true:

(1) If $\lambda \leq \mu+\alpha$, the steady state $u=0$ is locally asymptotically stable.

(2) If $\lambda>\mu+\alpha$, system (1.1) bifurcates from the trivial solution $u=0$ to an attractor $\Sigma_{\lambda}$.

(3) $\Sigma_{\lambda}$ is homeomorphic to $S^{1}$ and consists of exactly one cycle of steady solutions of (1.1).

(4) $\Sigma_{\lambda}$ can be expressed as

$$
\Sigma_{\lambda}=\{\tilde{x} \cos (x+\theta)+o(|\tilde{x}|) \mid \theta \in \mathbb{R}\},
$$

where $\tilde{x}=\sqrt{\frac{4(16 \mu+4 \alpha-\lambda)(\mu+\alpha-\lambda)}{3 a_{3}(16 \mu+4 \alpha-\lambda)+2 a_{2}^{2}}}\left(a_{2} \neq 0\right)$, or $\tilde{x}=\sqrt{\frac{4(\mu+\alpha-\lambda)}{3 a_{3}}}\left(a_{2}=0\right)$, and $\mu+\alpha<\lambda<\mu+\alpha+\epsilon, \epsilon$ is sufficiently small.

Proof of Theorem 4.1 We shall prove Theorem 4.1 in four steps.

Step 1. In this step, we study the eigenvalue problem of the linearized equation of (3.2) and find the eigenvectors and the critical value of $\lambda$. 
Consider the eigenvalue problem of the linear equation,

$$
L_{\lambda} u=\beta u
$$

It is not difficult to find that the eigenvalues and the normalized eigenvectors of (4.1) are

$$
\left\{\begin{array}{l}
\beta_{2 k-1}=\beta_{2 k}=\lambda-\mu k^{4}-\alpha k^{2}, \quad k=1,2, \ldots, \\
e_{2 k-1}=\frac{\sin k x}{\sqrt{\pi}}, \quad e_{2 k}=\frac{\cos k x}{\sqrt{\pi}},
\end{array}\right.
$$

under condition that we get the principle of exchange of stabilities

$$
\begin{aligned}
& \beta_{1}(\lambda)=\beta_{2}(\lambda) \begin{cases}<0, & \lambda<\mu+\alpha, \\
=0, & \lambda=\mu+\alpha, \\
>0, & \lambda>\mu+\alpha,\end{cases} \\
& \beta_{j}(\mu+\alpha)<0, \quad j \geq 3 .
\end{aligned}
$$

Step 2. We verify that for any $\lambda \in \mathbb{R}$, operator $L_{\lambda}+G$ satisfies conditions (2.2) and (2.3). Thanks to the results in $[9,18,19]$, we know that the operator $L_{\lambda}: H_{1} \rightarrow H$ is a sectorial operator which implies that condition (2.2) holds true.

It is easy to get the following inequality:

$$
\begin{aligned}
\|G(u)\|_{H}^{2} & =\int_{0}^{2 \pi}|g(u)|^{2} d x \\
& \leq C \int_{0}^{2 \pi}\left(\sum_{k=2}^{p}|u|^{2 k}\right) d x \\
& \leq C \sum_{k=2}^{p}\|u\|_{L^{2 k}(0,2 \pi)}^{2 k} \\
& \leq C \sum_{k=2}^{p}\|u\|_{H_{\frac{1}{2}}}^{2 k},
\end{aligned}
$$

which implies that $G(u)=o\left(\|u\|_{H_{\frac{1}{2}}}\right)$, where

$$
H_{\frac{1}{2}}=\left\{u \in H^{2}(0,2 \pi) \mid \int_{0}^{2 \pi} u d x=0, u(x+2 \pi)=u(x)\right\},
$$

then condition (2.3) holds true.

Step 3. In this part, we prove the existence of attractor bifurcation and analyze the topological structure of the attractor $\Sigma_{\lambda}$.

Let $E_{1}^{\lambda}=E_{0}=\operatorname{span}\left\{e_{1}, e_{2}\right\}, E_{2}^{\lambda}=E_{0}^{\perp}$. Let $\Phi$ be the center manifold function, in the neighborhood of $(u, \lambda)=(0, \mu+\alpha)$, we have

$$
u=y+\Phi(y)
$$

where $y=x_{1} e_{1}+x_{2} e_{2}$. 
Then the reduction equations of (3.2) are as follows:

$$
\left\{\begin{array}{l}
\frac{d x_{1}}{d t}=(\lambda-\mu-\alpha) x_{1}+\left\langle G(u), e_{1}\right\rangle \\
\frac{d x_{2}}{d t}=(\lambda-\mu-\alpha) x_{2}+\left\langle G(u), e_{2}\right\rangle
\end{array}\right.
$$

To get the exact form of the reduction equations, we need to obtain the expression of $\left\langle G(u), e_{1}\right\rangle$ and $\left\langle G(u), e_{2}\right\rangle$.

Let $G_{2}: H_{1} \times H_{1} \rightarrow H$ and $G_{3}: H_{1} \times H_{1} \times H_{1} \rightarrow H$ be the bilinear and trilinear operators of $G$ respectively, i.e.,

$$
\begin{aligned}
& G_{2}\left(u_{1}, u_{2}\right)=a_{2} u_{1} u_{2}, \\
& G_{3}\left(u_{1}, u_{2}, u_{3}\right)=a_{3} u_{1} u_{2} u_{3} .
\end{aligned}
$$

Since

$$
\left\langle G_{2}(y, y), e_{1}\right\rangle=\left\langle G_{2}(y, y), e_{2}\right\rangle=0
$$

the first order approximation of (4.3) does not work. Now, we shall find out the second order approximation of (4.3). And the most important of all is to obtain the approximation expression of the center manifold function.

By direct calculation, we have

$$
\left\langle G_{2}(y, y), e_{k}\right\rangle= \begin{cases}\frac{a_{2}}{\sqrt{\pi}} x_{1} x_{2}, & k=3, \\ \frac{a_{2}}{2 \sqrt{\pi}} x_{2}^{2}-\frac{a_{2}}{2 \sqrt{\pi}} x_{1}^{2}, & k=4, \\ 0, & k \neq 3,4 .\end{cases}
$$

According to the formula of Theorem 3.8 in [15] (or Remark 4.1), the center manifold function $\Phi$, in the neighborhood of $(u, \lambda)=(0, \mu+\alpha)$, can be expressed as

$$
\begin{aligned}
\Phi(y)= & -\sum_{k=3}^{\infty} \beta_{k}^{-1}\left\langle G_{2}(y, y), e_{k}\right\rangle e_{k}+O\left(\left(\left|\beta_{1}\right|^{2}+\left|\beta_{2}\right|^{2}\right)|y|^{2}\right)+o\left(|y|^{2}\right) \\
= & -(\lambda-16 \mu-4 \alpha)^{-1} \frac{a_{2}}{2 \sqrt{\pi}}\left(2 x_{1} x_{2} e_{3}+x_{2}^{2} e_{4}-x_{1}^{2} e_{4}\right) \\
& +O\left(|\lambda-\mu-\alpha|^{2}\left(\left|x_{1}\right|^{2}+\left|x_{2}\right|^{2}\right)\right)+o\left(\left|x_{1}\right|^{2}+\left|x_{2}\right|^{2}\right) .
\end{aligned}
$$

In the following, we calculate $\left\langle G(u), e_{j}\right\rangle, j=1,2$.

$$
\begin{aligned}
\left\langle G(u), e_{j}\right\rangle= & \left\langle G_{2}(y, \Phi(y)), e_{j}\right\rangle+\left\langle G_{2}(\Phi(y), y), e_{j}\right\rangle+\left\langle G_{3}(y, y, y), e_{j}\right\rangle \\
& +O\left(|\lambda-\mu-\alpha|^{2}\left(\left|x_{1}\right|^{3}+\left|x_{2}\right|^{3}\right)\right)+o\left(\left|x_{1}\right|^{3}+\left|x_{2}\right|^{3}\right), \quad j=1,2 .
\end{aligned}
$$

By direct calculation, we have

$$
\begin{aligned}
& \left\langle G_{2}(y, \Phi(y)), e_{1}\right\rangle \\
& \quad=\left\langle G_{2}(\Phi(y), y), e_{1}\right\rangle
\end{aligned}
$$




$$
\begin{aligned}
&=-(\lambda-16 \mu-4 \alpha)^{-1} \frac{a_{2}^{2}}{4 \pi} x_{1}^{3}-(\lambda-16 \mu-4 \alpha)^{-1} \frac{a_{2}^{2}}{4 \pi} x_{1} x_{2}^{2} \\
&+O\left(|\lambda-\mu-\alpha|^{2}\left(\left|x_{1}\right|^{3}+\left|x_{2}\right|^{3}\right)\right)+o\left(\left|x_{1}\right|^{3}+\left|x_{2}\right|^{3}\right), \\
&\left\langle G_{2}(y, \Phi(y)), e_{2}\right\rangle \\
&=\left\langle G_{2}(\Phi(y), y), e_{2}\right\rangle \\
&=-(\lambda-16 \mu-4 \alpha)^{-1} \frac{a_{2}^{2}}{4 \pi} x_{2}^{3}-(\lambda-16 \mu-4 \alpha)^{-1} \frac{a_{2}^{2}}{4 \pi} x_{1}^{2} x_{2} \\
&+O\left(|\lambda-\mu-\alpha|^{2}\left(\left|x_{1}\right|^{3}+\left|x_{2}\right|^{3}\right)\right)+o\left(\left|x_{1}\right|^{3}+\left|x_{2}\right|^{3}\right), \\
&\left\langle G_{3}(y, y, y), e_{1}\right\rangle=\frac{3 a_{3}}{4 \pi} x_{1}^{3}+\frac{3 a_{3}}{4 \pi} x_{1} x_{2}^{2}, \\
&\left\langle G_{3}(y, y, y), e_{2}\right\rangle=\frac{3 a_{3}}{4 \pi} x_{2}^{3}+\frac{3 a_{3}}{4 \pi} x_{1}^{2} x_{2},
\end{aligned}
$$

then we obtain the expression of $\left\langle G(u), e_{j}\right\rangle, j=1,2$.

$$
\begin{aligned}
& \left\langle G(u), e_{1}\right\rangle=A x_{1}^{3}+A x_{1} x_{2}^{2}+O\left(|\lambda-\mu-\alpha|^{2}\left(\left|x_{1}\right|^{3}+\left|x_{2}\right|^{3}\right)\right)+o\left(\left|x_{1}\right|^{3}+\left|x_{2}\right|^{3}\right), \\
& \left\langle G(u), e_{2}\right\rangle=A x_{1}^{2} x_{2}+A x_{2}^{3}+O\left(|\lambda-\mu-\alpha|^{2}\left(\left|x_{1}\right|^{3}+\left|x_{2}\right|^{3}\right)\right)+o\left(\left|x_{1}\right|^{3}+\left|x_{2}\right|^{3}\right),
\end{aligned}
$$

where $A=-(\lambda-16 \mu-4 \alpha)^{-1} \frac{a_{2}^{2}}{2 \pi}+\frac{3 a_{3}}{4 \pi}$.

Putting (4.4) into (4.3), we have the reduction equations

$$
\left\{\begin{aligned}
\frac{d x_{1}}{d t}= & (\lambda-\mu-\alpha) x_{1}+A x_{1}^{3}+A x_{1} x_{2}^{2} \\
& +O\left(|\lambda-\mu-\alpha|^{2}\left(\left|x_{1}\right|^{3}+\left|x_{2}\right|^{3}\right)\right)+o\left(\left|x_{1}\right|^{3}+\left|x_{2}\right|^{3}\right), \\
\frac{d x_{2}}{d t}= & (\lambda-\mu-\alpha) x_{2}+A x_{1}^{2} x_{2}+A x_{2}^{3} \\
& +O\left(|\lambda-\mu-\alpha|^{2}\left(\left|x_{1}\right|^{3}+\left|x_{2}\right|^{3}\right)\right)+o\left(\left|x_{1}\right|^{3}+\left|x_{2}\right|^{3}\right) .
\end{aligned}\right.
$$

For the case of $\lambda<\mu+\alpha$, it is obvious that $u=0$ is locally asymptotically stable. For the case of $\lambda=\mu+\alpha$, if $2 a_{2}^{2}+(45 \mu+9 \alpha) a_{3}<0$, which implies that $A<0$, then $u=0$ is also locally asymptotically stable. Assertion (1) of Theorem 4.1 is proved.

Since the following equality holds true:

$$
x_{1}\left(A x_{1}^{3}+A x_{1} x_{2}^{2}\right)+x_{2}\left(A x_{1}^{2} x_{2}+A x_{2}^{3}\right)=A\left(x_{1}^{2}+x_{2}^{2}\right)^{2},
$$

according to Theorems 2.1, 2.2 and Remark 2.1, we can conclude that if $\lambda>\mu+\alpha$, equation (1.1) bifurcates from $u=0$ to an attractor $\Sigma_{\lambda}$, which is homeomorphic to $S^{1}$.

Step 4. In the last step, we show that the bifurcated attractor of (3.2) consists of a singularity cycle.

Since the even function space is an invariant subspace of $L_{\lambda}+G$ defined by (3.1), we shall consider the bifurcation problem in the even function space and prove that system (1.1) bifurcates from $(u, \lambda)=(0, \mu+\alpha)$ to two steady solutions. For any function $v$ in the even function space can be expressed as follows:

$$
v=\sum_{k \geq 1} x_{2 k} e_{2 k}
$$


by the Lyapunov-Schmidt reduction method used in Step 3, we can deduce that the reduction equation of (1.1) is as follows:

$$
\frac{d x_{2}}{d t}=(\lambda-\mu-\alpha) x_{2}+A x_{2}^{3}+O\left(|\lambda-\mu-\alpha|^{2}\left|x_{2}\right|^{3}\right)+o\left(\left|x_{2}\right|^{3}\right),
$$

which implies that (1.1) bifurcates from $(u, \lambda)=(0, \mu+\alpha)$ to two steady solutions $V_{\lambda}^{ \pm}(x, t)=$ $\pm \sqrt{\frac{4(16 \mu+4 \alpha-\lambda)(\mu+\alpha-\lambda)}{3 a_{3}(16 \mu+4 \alpha-\lambda)+2 a_{2}^{2}}} \cos x+$ h.o.t. in the space of even functions.

Since the solutions of (2.1) are translation invariant,

$$
V_{\lambda}^{+}(x, t) \rightarrow V_{\lambda}^{+}(x+\theta, t), \quad \forall \theta \in \mathbb{R}
$$

the set

$$
T=\left\{V_{\lambda}^{+}(x+\theta, t) \mid \theta \in \mathbb{R}\right\}
$$

represents $S^{1}$ in $H_{1}$, which implies that $\sum_{\lambda}$ consists of exactly one circle of steady solutions of (1.1). This completes the proof of Theorem 4.1.

Remark 4.1 Suppose that $\left\{e_{i}\right\}$, the generalized eigenvectors of $L_{\lambda}$, form a basis of $H$ with the dual basis $\left\{e_{i}^{*}\right\}$ such that

$$
\left\langle e_{i}, e_{j}^{*}\right\rangle_{H} \begin{cases}=0, & \text { if } i \neq j, \\ \neq 0, & \text { if } i=j .\end{cases}
$$

We have

$$
\begin{aligned}
& v=x+y \in E_{1}^{\lambda} \oplus E_{2}^{\lambda}, \\
& x=\sum_{i=1}^{m} x_{i} e_{i} \in E_{1}^{\lambda}, \\
& y=\sum_{i=m+1}^{\infty} x_{i} e_{i} \in E_{2}^{\lambda} .
\end{aligned}
$$

Then near $\lambda=\lambda_{0}$, the center manifold function $\phi(x, \lambda)$ in Theorem 3.8 in [15] can be expressed as follows:

$$
\phi(x, \lambda)=\sum_{j=m+1}^{\infty} \phi_{j}(x, \lambda) e_{j}+O\left(|\operatorname{Re} \beta(\lambda)| \cdot\|x\|^{k}\right)+o\left(\|x\|^{k}\right),
$$

where

$$
\phi_{j}(x, \lambda)=-\frac{1}{\beta_{j}(\lambda)}\left\langle G_{k}(x, \ldots, x), e_{j}^{*}\right\rangle_{H} .
$$

Remark 4.2 If $g(s)$ in (1.1) is not a polynomial but a $C^{\omega}$ with Taylor's expansion in $s=0$ as $g(s)=\sum_{k=2}^{\infty} a_{k} s^{k} ;$ if $2 a_{2}^{2}+45 \mu a_{3}+9 \alpha a_{3}<0$ is satisfied, then the conclusions of Theorem 4.1 also hold true. 
Remark 4.3 If the higher order terms $\sum_{k=4}^{p} a_{k} u^{k}$ in $g(u)$ are omitted, from the proof of Theorem 4.1, it is easy to see that the conclusions of Theorem 4.1 also hold true.

\section{Applications}

In this section, we apply Theorem 4.1 to a model of the population density for singlespecies as follows:

$$
\left\{\begin{array}{l}
\frac{\partial v}{\partial t}=-\mu \frac{\partial^{4}}{\partial x^{4}} v+\alpha \frac{\partial^{2}}{\partial x^{2}} v+b_{1} v+b_{2} v^{2}+a_{3} v^{3}+b_{0}, \quad(x, t) \in \mathbb{R} \times(0, \infty) \\
\int_{0}^{2 \pi} v(x, t) d x=\frac{a_{2}}{2 a_{3}} \pi, \quad t \geq 0 \\
v(x, t)=v(x+2 k \pi, t), \quad \forall k \in \mathbb{Z} \\
v(x, 0)=u_{0}+v_{0}, \quad x \in \mathbb{R}
\end{array}\right.
$$

where $\mu, \alpha$ are the diffusion coefficients, $\nu$ is the population density for single-species, and $a_{2}<0, a_{3}<0, b_{0}=-\lambda \frac{a_{2}}{4 a_{3}}+\frac{3}{64} \frac{a_{2}^{3}}{a_{3}^{2}}, b_{1}=\lambda-\frac{5}{16} \frac{a_{2}^{2}}{a_{3}}, b_{2}=\frac{a_{2}}{4}$. It is easy to see that $b_{0}<0, b_{1}>0$ and $b_{2}<0$. Inspired by the work of Murray [20], $b_{1}$ represents the birth rate, $b_{2} v^{2}+a_{3} v^{3}$ describes the intra specific competition, and $b_{0}$ stands for the emigration which arises from disease.

It is not difficult to verify that $v_{0}=\frac{a_{2}}{4 a_{3}}$ is a positive steady solution of system (5.1). From the translation

$$
u(x, t)=v(x, t)-v_{0}
$$

we derive the following system:

$$
\left\{\begin{array}{l}
\frac{\partial u}{\partial t}=-\mu \frac{\partial^{4}}{\partial x^{4}} u+\alpha \frac{\partial^{2}}{\partial x^{2}} u+\lambda u+a_{2} u^{2}+a_{3} u^{3}, \quad(x, t) \in \mathbb{R} \times(0, \infty), \\
\int_{0}^{2 \pi} u(x, t) d x=0, \quad t \geq 0, \\
u(x, t)=u(x+2 k \pi, t), \quad \forall k \in \mathbb{Z}, \\
u(x, 0)=u_{0}, \quad x \in \mathbb{R} .
\end{array}\right.
$$

According to Remark 4.3, if the condition $2 a_{2}^{2}+45 \mu a_{3}+9 \alpha a_{3}<0$ is satisfied, the conclusions of Theorem 4.1 for system (5.3) also hold true. Consequently, from the translation (5.2), we have the following results for (5.1).

Theorem 5.1 For problem (5.1), if $2 a_{2}^{2}+45 \mu a_{3}+9 \alpha a_{3}<0$ is satisfied, then the following assertions hold true:

(1) If $b_{1} \leq \mu+\alpha-\frac{5}{16} \frac{a_{2}^{2}}{a_{3}}$, the steady state $v_{0}=\frac{a_{2}}{4 a_{3}}$ is locally asymptotically stable (Figure 1).

(2) If $b_{1}>\mu+\alpha-\frac{5}{16} \frac{a_{2}^{2}}{a_{3}}$, system (5.1) bifurcates from the solution $v_{0}$ to an attractor $\Sigma_{b_{1}}$. This implies that the stability will switch from the original state (i.e., $\left.v_{0}\right)$ to a new one (i.e., $\Sigma_{b_{1}}$ ) (Figure 1).

(3) $\Sigma_{b_{1}}$ is homeomorphic to $S^{1}$ and consists of exactly one cycle of steady solutions of (5.1) (Figure 1). 
Figure 1 Bifurcation diagram for the model of the population density for single-species. (1) Bifurcation appears at $\lambda_{0}=\mu+\alpha$ $-\frac{5}{16} \frac{a_{2}^{2}}{a_{3}}$. (2) Bifurcated attractor $\Sigma_{b_{1}}$ is the boundary of the shaded region. (3) The first horizontal solid line from above denotes that the solution $v=v_{0}$ is stable, and the horizontal dotted line means this solution is unstable.

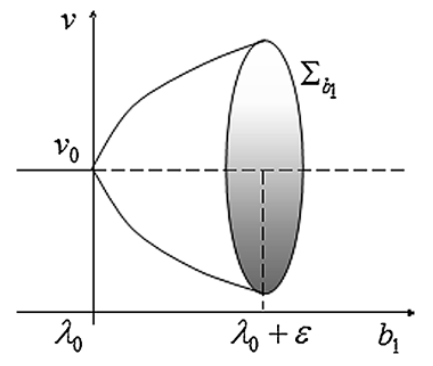

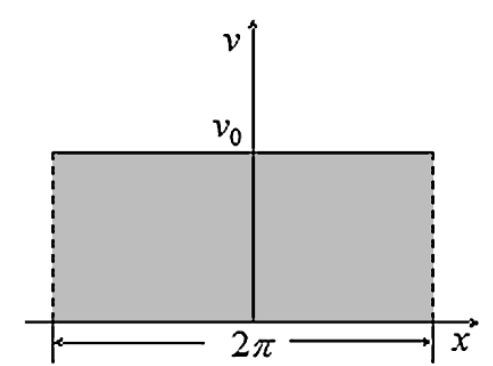

A

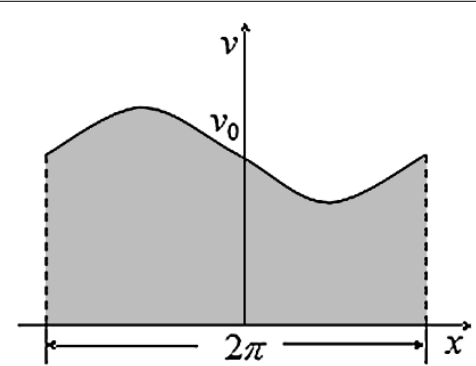

B

Figure 2 The spatial distribution of the population density. (1) Figure 2(A) shows that the population density keeps a uniform spatial distribution when the birth rate is low. (2) Figure 2(B) shows that the population density changes periodically with space when the birth rate becomes high enough. (3) The area of the shaded regions stands for the population of this single-species. And the area of the shaded region in Figure 2(A) is equal to the area of the shaded region in Figure 2(B).

(4) $\Sigma_{b_{1}}$ can be expressed as

$$
\Sigma_{b_{1}}=\left\{v_{0}+\tilde{x} \cos (x+\theta)+o(|\tilde{x}|) \mid \theta \in \mathbb{R}\right\},
$$

where $\tilde{x}=\sqrt{\frac{4(16 \mu+4 \alpha-\lambda)(\mu+\alpha-\lambda)}{3 a_{3}(16 \mu+4 \alpha-\lambda)+2 a_{2}^{2}}}$, and $\mu+\alpha<\lambda<\mu+\alpha+\epsilon, \epsilon$ is sufficiently small.

Furthermore, Theorem 5.1 and the equality

$$
\int_{0}^{2 \pi} v(x, t) d x=\frac{a_{2}}{2 a_{3}} \pi, \quad t \geq 0
$$

yield the following biological results.

Biological results For the model (5.1), if $2 a_{2}^{2}+45 \mu a_{3}+9 \alpha a_{3}<0$ is satisfied, we have the following assertions:

(1) The population of this single-species is a conservative quantity.

(2) If the birth rate is low, then the population density will keep a uniform spatial distribution (Figure 2(A)).

(3) If the birth rate becomes high enough, then the spatial distribution of the population density will not keep uniform but change periodically with space (Figure 2(B)).

\section{Discussion}

Taking $\alpha=1, \lambda=1, g(u)=-u^{3}$ in (1.1), Peletier and Troy [6] analyzed stationary antisymmetric single-bump periodic solutions. They found that the coefficient of the fourth-order 
derivative term $\mu$ played a role of system parameter. If $\mu \leq \frac{1}{8}$, the family of periodic solutions is still very similar to that of the Fisher-Kolmogorov equations. However, if $\mu>\frac{1}{8}$, different families of periodic solutions emerged.

Taking $\mu=1, \lambda=1$ in (1.1), and under hypothesis that $g(1)=-1, g^{\prime}(1)<-1, g^{\prime}(u)<0$ for $0<u<1$, Rottschäer and Wayne [8] showed that for every positive wavespeed, there exists a traveling wave. And they also found that there exists a critical wavespeed $c^{*}$. If $c \geq c^{*}$, the solution is monotonic; otherwise, the solution is oscillatory.

Unlike the work mentioned above, which focuses on the structure of solutions varying with the system parameter ( $\mu$ or $c$ ), the manuscript presented here investigates the topological structure and the stability of solutions varying with the system parameter, i.e., $\lambda$. Firstly, if $\lambda \leq \mu+\alpha$, the bifurcated attractor consists of the trivial solution; if $\lambda>\mu+\alpha$, the bifurcated attractor consists of only one cycle of steady state solutions and is homeomorphic to $S^{1}$. Secondly, if $\lambda \leq \mu+\alpha$, the trivial solution is locally asymptotically stable. However, if $\lambda>\mu+\alpha$, the stability switches from the trivial solution to the bifurcated attractor.

Since the increment of dimension of spatial domain may lead to much richer bifurcated behavior, further investigation on higher dimension of spatial domain is necessary in the future.

\section{Conclusions}

In this article, we first prove the existence of attractor bifurcation when the system parameter crosses critical number $\mu+\alpha$, which is the first eigenvalue of the eigenvalue problem of the linearized equation of (1.1). Second, we show that the stability of solutions varies with the system parameter $\lambda$. If $\lambda \leq \mu+\alpha$, the trivial solution $u=0$ is locally asymptotically stable. However, if $\lambda>\mu+\alpha$, the stability switches from $u=0$ to $\Sigma_{\lambda}$. Third, the topological structure of the attractor is investigated. We prove that the attractor $\Sigma_{\lambda}$ consists of only one cycle of steady state solutions and is homeomorphic to $S^{1}$. At last, the expression of bifurcated solution is also obtained.

\section{Competing interests \\ The authors declare that they have no competing interests.}

Authors' contributions

Both authors read and approved the final manuscript.

\section{Author details}

${ }^{1}$ College of Computer Science, Civil Aviation Flight University of China, Guanghan, Sichuan 618307, P.R. China. ${ }^{2}$ College of Mathematics and Software Science, Sichuan Normal University, Chengdu, Sichuan 610066, P.R. China.

\section{Acknowledgements \\ The authors are grateful to the anonymous referees whose careful reading of the manuscript and valuable comments were very helpful for revising and improving our work.}

Received: 24 October 2012 Accepted: 5 July 2013 Published: 19 July 2013

References

1. Coullet, P, Elphick, C, Repaux, D: Nature of spatial chaos. Phys. Rev. Lett. 58, 431-434 (1987)

2. Dee, G, Saarloose, W: Bistable systems with propagating fronts leading to pattern formation. Phys. Rev. Lett. 60 , 2641-2644 (1988)

3. Zimmermann, W: Propagating fronts near a Lipschitz point. Phys. Rev. Lett. 66, 1546 (1991)

4. Kalies, WD, van der Vorst, RCAM: Multitransition homoclinic and heteroclinic solutions of the extended Fisher-Kolmogorov equation. J. Differ. Equ. 131, 209-228 (1996)

5. Tersian, S, Chaparova, J: Periodic and homoclinic solutions of extended Fisher-Kolmogorov equations. J. Math. Anal. Appl. 260, 490-506 (2001) 
6. Peletier, LA, Troy, WC: Spatial patterns described by the extended Fisher-Kolmogorov equation: periodic solutions. SIAM J. Math. Anal. 28, 1317-1353 (1997)

7. van den Berg, JB: The phase-plane picture for a class of fourth-order conservative differential equations. J. Differ. Equ. $161,110-153$ (2000)

8. Rottschäer, V, Wayne, CE: Existence and stability of traveling fronts in the extended Fisher-Kolmogorov equation. J. Differ. Equ. 176, 532-560 (2001)

9. Luo, H, Zhang, Q: Regularity of global attractor for the fourth-order reaction-diffusion equation. Commun. Nonlinear Sci. Numer. Simul. 17, 3824-3831 (2012)

10. Kwapisz, J: Uniqueness of the stationary wave for the extended Fisher-Kolmogorov equation. J. Differ. Equ. 165, 235-253 (2000)

11. Bartuccelli, MV: On the asymptotic positivity of solutions for the extended Fisher-Kolmogorov equation with nonlinear diffusion. Math. Methods Appl. Sci. 25, 701-708 (2002)

12. Benguria, RD, Depassier, MC: On the transition from pulled to pushed monotonic fronts of the extended Fisher-Kolmogorov equation. Physica A 356, 61-65 (2005)

13. Peletier, LA, Troy, WC: A topological shooting method and the existence of kinks of the extended Fisher-Kolmogorov equation: periodic solutions. Topol. Methods Nonlinear Anal. 6, 331-355 (1995)

14. Ma, T, Wang, SH: Attractor bifurcation theory and its applications to Rayleigh-Bénard convection. Commun. Pure Appl. Anal. 2, 591-599 (2003)

15. Ma, T, Wang, SH: Bifurcation Theory and Applications. Nonlinear Science Series A, vol. 53. World Scientific, Singapore (2005)

16. Park, J: Bifurcation and stability of the generalized complex Ginzburg-Landau equation. Commun. Pure Appl. Anal. 7(5), 1237-1253 (2008)

17. Zhang, YD, Song, LY, Axia, W: Dynamical bifurcation for the Kuramoto-Sivashinsky equation. Nonlinear Anal. 74(4), 1155-1163 (2011)

18. Temam, R: Infinite-Dimensional Dynamical Systems in Mechanics and Physics. Appl. Math. Sci., vol. 68. Springer, New York (1997)

19. Pazy, A: Semigroups of Linear Operators and Applications to Partial Differential Equations. Appl. Math. Sci., vol. 44. Springer, New York (2006)

20. Murray, JD: Mathematical Biology. Springer, New York (1989)

doi:10.1186/1687-2770-2013-169

Cite this article as: Zhang and Luo: Attractor bifurcation for the extended Fisher-Kolmogorov equation with periodic boundary condition. Boundary Value Problems 2013 2013:169.

\section{Submit your manuscript to a SpringerOpen ${ }^{\circ}$ journal and benefit from:}

- Convenient online submission

Rigorous peer review

- Immediate publication on acceptance

- Open access: articles freely available online

- High visibility within the field

- Retaining the copyright to your article 